\title{
Conditional penalty of the emotions towards the skin stressed genesis of the acnae, hyperalgesiae and pruritus Violeta Popovska*
}

\author{
Address: Medical Centre in Kumanovo, F.Y.R.O.M
}

* Corresponding author

from International Society on Brain and Behaviour: 2nd International Congress on Brain and Behaviour Thessaloniki, Greece. 17-20 November 2005

Published: 28 February 2006

Annals of General Psychiatry 2006, 5(SuppI I):S293 doi:I0.I I86/I744-859X-5-SI-S293

\begin{abstract}
Background
The psychical suffering of the man is not just his secret experience - the mental apparatus is a cruel judge who does not have compassion for the convicted, who cries, fears, gets angry and repels by his own reflection in the mirror.
\end{abstract}

The stressful condition is a universal pathogenic factor or co-factor of all the morbid processes in the organism. The human skin is one of the chains of exponents of the superiority of the psyche to the body. All the consecutives of the skin and its annexes content identical receptive and biochemical scheme the same as the brain. The near contact of the skin and psyche is realised neuronally (A $\beta, \mathrm{A} \mathrm{P}$ and $\mathrm{C}$ nerve fibres) or humerally. The realisation of the interplay is on the level of Merkel and Langer Hans' cells, mastocytes, keratinocytes, fibroblasts, melanocytes etc.

The focus of this study's interest is the psychological conditionality of the acnae and the phenomenon of hyperalgesiae and pruritus in a situation of perceptive stress.

The acnes are chronicle and inflamed illness of the pylosebaceous unit, which are normally manifested in the period of adolescence. According to the latest facts, the secretion of the sebum only depends on the concentration and utilisation of the androgynous substances from suprarenal background. During a period of a long stressful situation, in the reaction of the organism two hyperactive axes are interlaced:The first: corticotrophin - with an intense release of proopiomelanocortic substratum (ACTH, alpha MSH, beta endolfin, beta lipocortin and a little dose of androgenic active compounds) and the second: adrenomedular (with an increased activity of the biogenic amines - NA, DA, Adr., a.holin), neuropeptides, peptides and neuromodulators. These released sub- stances have a bifocal effect - they determine the emotional, cognitive and behavioural schemes of the persona but they simultaneously correspondent with their receptors on the level of the skin.

The relevant fascinated factors for the appearance of acnes are androgenic, ACTH, STH, MSN, GnRH, estrogenic, progesterone, NA, DA, Adr., GABA, corticosteroid - which are "stressful substances". An exceptional part takes the factors from and towards the keratinocytes and melanocytes (through the receptive web of their surface for the supst. P, VIP, MSH, a.holin, estrogens, progesterone and ACTH). The whole multi-way communicational web results with the appearance of sebum.

Mutual protagonists of the brain and the skin during the appearance of the psychogenic hyperalgesiae and pruritus are 5-ht, Histamine, supst. P, VIP, endogenic drugs, neurotensin, prostaglandins and bradicinines. Some of them have a direct impact, and the rest of them indirect with histaminoliberation. For example, in a depressive situation there is a decline of 5-ht and an increase of production of the supst. P (from the dorsal parts of m.spinalis) and that lead to a histamine release of mastocytes, increased production of IL-2, intensive production of eicosanoids, hyperdinia, pruritus etc.

\section{Materials and methods}

In a period of two years, 29 patients (18 female and 11 male) between the ages of 19-38 were treated in our hospital. The psychological battery of tests were used, for example, MMPI, NEOPIR, Mahover, lifestyle by whose greater or lesser exceptional variables a zone for hiding certain personal features was reached: high level of vulnerability, low level of impulsiveness, medium level of anxiety, depressiveness and hostility, friendliness, 
assertiveness, imagination, toleration, low level of action, quest for excitement, altruism, modesty, self-discipline and duty. They were treated with cognitive and behavioural therapy, relaxation techniques, method of investigation and suggestive theory.

\section{Results}

After the application of the psycho medicaments (Diazepam, Loram) and anti depressives (Doxepin and Maprotilin) in a combination with psychotherapeutic intervention, all the patients normalised their skin sensibility in a relatively fast period of time ( 2 to 11 days). Hyperalgesiae appeared as a more resistant symptom than pruritus. The acne phenomenon remained for 2 to 2 and half months, in addition 21 patients completely normalised the production of the oil glands. As an aggravated factor in accord with the psychotherapeutic efficiency a high level of narcotics was found (7 patients) as well as their tendency to addiction (11 patients). Their intellectual level showed as a successful factual tool (9 of the patients had IQ 115 to 121). Their living conditions were a modifying factor -16 of them were working, 3 of them single, 8 of them lived in a rented house, one of them had 5 children and was not able to give the two of them a further education. The patients with a higher level of friendliness, assertiveness, action, lower hostility and not that modest fantasy clinically developed more discreet skin defects with less Dynic feeling (the treatment of them was faster and more successful).

\section{Discussion}

The psychological profile of this group of patients, besides the significantly increased sphere of similarity, is somewhat more hetero genus in accord with the relative uniformity of type A and B. Because of its complexity, the stressful psychological reaction mobilises, to full capacity, all the defensive resources of the personalities, which complicates the map of variations of the mental excitements and reactions. On the other hand, the skin is a diametrical exterior of the intimae, so the roads between them are very long and complicated. That is why we cannot establish a single predicative formula of the skin manifestations. The principle of treatment "outer" is just temporarily makes corrections to the manifested result of this process without the possibility to its eradication. Selfcorrection in psychological frame and its opinion about self and reality is the real ad integrum therapeutic solution.

The three phases of the stressful condition - alarm, defence and exhaust are quite different psychic, biochemical and skin manifestations. The phase of alarm (activation of the reflexes for fight or escape, reflex of deadness or rational calmness) would basically follow the line of the amine brain activation (NA, Adr., DA, a.holine). The second phase of defence (with the activation of the defensive psychological mechanisms in accord with the individual personal organisation and adaptive capacities) would follow the chemistry of RF and RH factors from HyTH and $\mathrm{HyFz}$, with an increased activeness of the hormones, neuropeptides, peptides and the appearance of certain skin changes. The third phase - exhaust (with the development of the psychic changes) includes the development of the complex web of interactions of the released "stressful substances".

In this case the more significant is the correlation between the ACTH-endogenic drugs and endogenic drugs supst.P, cortisol-testosteron, PRL-5-alfa reduction, 5-HTprostaglandine and encephalin - prolactine. 